\title{
Oxidation Kinetics of Pitch Based Carbon Fibers
}

\author{
Jae-Seung Roh^ \\ School of Advanced Materials and System Engineering, Kumoh National Institute of Technology 1, Yangho-dong, Gumi, Gyungbuk 730-701, Korea \\ •e-mail: jsroh@kumoh.ac.kr \\ (Received May 2, 2008; Accepted June 4, 2008)
}

\begin{abstract}
High modulus pitch based carbon fibers (HM) were exposed to isothermal oxidation using tube furnace in carbon dioxide gas to study the oxidation kinetics under the temperature of $800-1100^{\circ} \mathrm{C}$. The kinetic equation $f=1-\exp \left(-a t^{b}\right)$ was introduced and the constant $b$ was obtained in the range of 1.02 1.42. The oxidation kinetics were evaluated by the reaction-controlling regime (RCR) depending upon the apparent activation energies with the conversion increasing from 0.2 to 0.8 . The activation energies decrease from 24.7 to $21.0 \mathrm{kcal} / \mathrm{mole}$ with the conversion increasing from 0.2 to 0.8 , respectively. According to the $\mathrm{RCR}$, the reaction was limited by more diffusion controlling regime for the HM fibers with the conversion increasing. Therefore, it seems that the oxidation which is under the diffusion controlling regime takes place continuously from the skin to the core of the fiber.
\end{abstract}

Keywords : Oxidation, Kinetics, Mechanism, High modulus carbon fiber.

\section{Introduction}

Oxidation reaction of carbon based materials take place at the active sites preferentially. The other factors affecting characteristics of oxidation reaction include microstructural characteristics related to the inhomogeneity taking place during the manufacturing of the carbon materials, and structural changes of pores and of carbon materials changed by oxidation reaction. Since the reaction mechanism alternates according to the model interpreting reaction and experimental facilities, the reaction mechanism much more complicated [1-5].

Most researchers explain the activation mechanism by a reaction-controlling regime after gaining activation energy when they measured the average rate of the overall activation of gasification reaction [6-11]. However, the report on interpretation of reaction mechanism of activated carbon materials via the data of activation energy with a tendency is hardly found. There are some reports only on granular carbon materials, but not the interpretation of reaction mechanism related to oxidation of activated carbon fiber is easily found.

The purpose of this study is that the clear explanation on oxidation reaction mechanism of high modulus carbon fibers(HM) was tried by comparing the result of isotropic pitch based carbon fibers(IP) reported earlier $[12,13]$.

\section{Experimental}

High modulus mesophase pitch based carbon fibers(HM-
60, called HM) and isotropic pitch based carbon fibers (Kureha T-101S, called IP) were used as raw materials. Based on assortment of carbon fibers according to crystallites orientation, IP fiber is manufactured around $1000^{\circ} \mathrm{C}$ and is isotropic carbon fiber with disordered crystallites orientation, and HM fiber is a highly strengthened carbon fiber with modulus $584 \mathrm{GPa}$.

Fig. 1 and Fig. 2 show SEM photographs of original fibers. HM fibers have been exposed to air for two hours at $400^{\circ} \mathrm{C}$ and surface protecting material was removed, still show $0 \%$ of weight loss. HM fiber has the texture along the fiber length shown in Fig. 2. As in the photograph the shape of IP fiber is an ellipse and its surface is smooth with no texture. The physical properties of the raw fibers were summarized in Table 1.

The original fiber has been activated by isothermal oxidation in the horizontal tube reactor by carbon dioxide gas at $800^{\circ} \mathrm{C}, 900^{\circ} \mathrm{C}, 1,000^{\circ} \mathrm{C}$, and $1,100^{\circ} \mathrm{C}$. Burn-off was calculated by dividing the weight loss after activation by original weight of sample. The detailed oxidation reaction method of the fibers and measurement of specific surface area(SSA) are the same as reported earlier[12,13].

\section{Results and Discussion}

\subsection{Isothermal oxidation reaction and SSA}

Fig. 3 represents the relation between the weight changes of the original fiber in carbon dioxide and reaction time. As the temperature of oxidation reaction gets higher, the time 

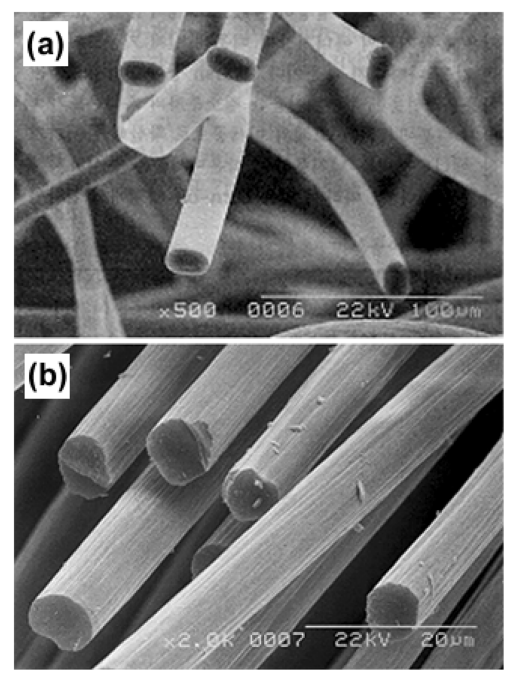

Fig. 1. SEM photographs of original IP fibers (a) and desized original HM fibers (b).
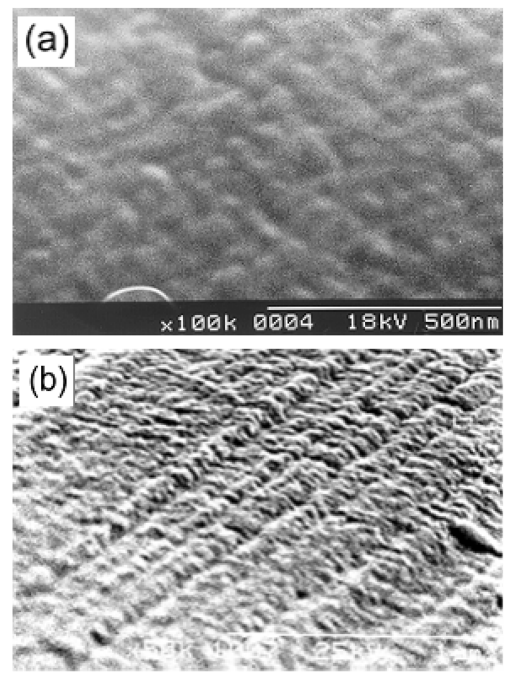

Fig. 2. Highly magnified SEM photographs of original IP fiber $(\times 100,000)(a)$ and original HM fiber $(\times 50,000)(b)$.

for weight loss rapidly decreased. Also, oxidation reaction of HM fiber proceeds very slowly compared to IP fiber at same temperature reported earlier [12].

Table 2 shows the SSA of oxidized fibers. Oxidized HM fibers show a very small SSA value compared to IP fiber, and present higher SSA oxidized at $1100^{\circ} \mathrm{C}$ than oxidized at $900^{\circ} \mathrm{C}$. Oxidized IP fibers show very high SSA as it was oxidized at $900^{\circ} \mathrm{C}$ and $1000^{\circ} \mathrm{C}$.
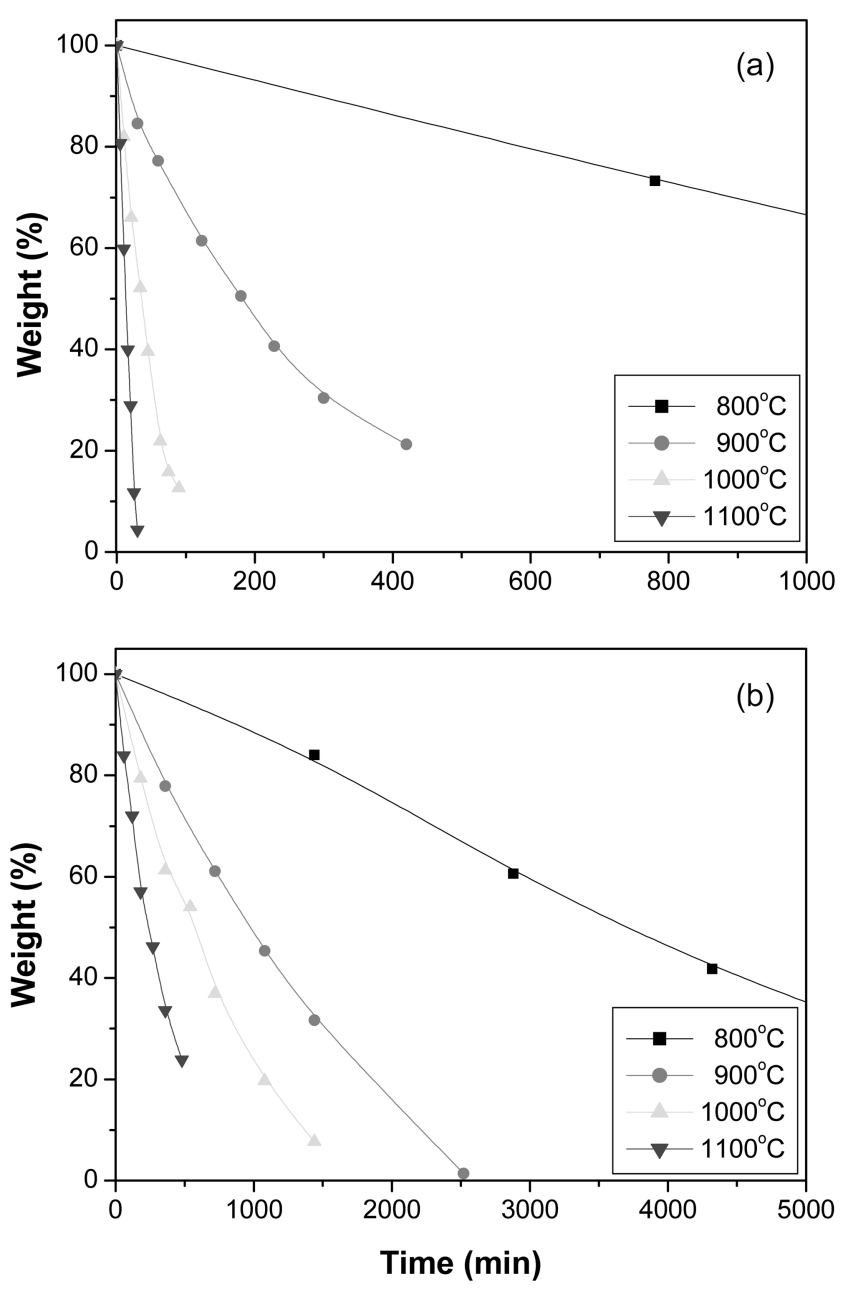

Fig. 3. Weight changes as a function of oxidation time in carbon dioxide gas of IP fibers (a) and HM fibers (b).

Fig. 4 and Fig. 5 present SEM images show a large difference between before and after oxidation of IP fibers and HM fibers. Fig. 4 shows the images of IP fiber that was burnoffed to $49 \%$ and $79 \%$ at $900^{\circ} \mathrm{C}$ but it don't show a large difference in surface morphology compared to the original fibers as shown in Fig. 2(a). It is believed that pores well developed in activated IP fiber from the SEM pictures, and most of them are micropores.

Fig. 5 show the SEM images of oxidized HM fibers with as similar weight losses at 800,900 and $1100^{\circ} \mathrm{C}$. The surface of original HM fibers show very well developed texture but no pores as shown in Fig. 2(b). Fig. 5(a) is the surface of 47 $\%$ burn-offed at $800^{\circ} \mathrm{C}$ showing macropores of over few

Table 1. Physical Properties of the Raw Fibers

\begin{tabular}{cccccc}
\hline Fibers & Filament Diameter $(\mu \mathrm{m})$ & Tensile Strength $(\mathrm{GPa})$ & Modulus $(\mathrm{GPa})$ & Elongation $(\%)$ & Density $\left(\mathrm{g} / \mathrm{cm}^{3}\right)$ \\
\hline HM & 7.0 & 2.94 & 584 & 0.5 & 2.2 \\
$\mathrm{IP}$ & 14.5 & 0.72 & 32 & 2.2 & 1.65 \\
\hline
\end{tabular}


Table 2. Specific Surface Areas(SSAs) of Oxidized Fibers

\begin{tabular}{cccc}
\hline Fibers & $\begin{array}{c}\text { Temperature } \\
\left({ }^{\circ} \mathrm{C}\right)\end{array}$ & $\begin{array}{c}\text { Burn-off } \\
(\%)\end{array}$ & $\begin{array}{c}\text { SSA } \\
\left(\mathrm{m}^{2} / \mathrm{g}\right)\end{array}$ \\
\hline \multirow{3}{*}{$\mathrm{HM}$} & 900 & 55 & 72 \\
\hline \multirow{2}{*}{$\mathrm{IP}$} & 1100 & 54 & 110 \\
\cline { 2 - 4 } & 800 & 47 & 1,825 \\
\cline { 2 - 4 } & 900 & 49 & 3,072 \\
\cline { 2 - 4 } & 1000 & 48 & 2,236 \\
\hline
\end{tabular}

hundreds of nm were created. Fig. 5(b) that had 51\% burnoffed at $900^{\circ} \mathrm{C}$ has many pores on the surface which are mesopores from tens to hundreds of nm that are smaller than oxidized at $800^{\circ} \mathrm{C}$. Fig. 5(c) shows $50 \%$ burn-offed at $1100^{\circ} \mathrm{C}$, whose surface is smooth and whose pores seem to be very small.

As such, considering the fact that pores on the SSA are different according to oxidizing temperatures, oxidation procedures are expected to be different according to oxidizing temperatures as well.

\subsection{Kinetic equation}

Oxidation rates were calculated by exponential function. Conversion $f$ can be expressed $f=1-\frac{W}{W_{0}}$, and the kinetic equation $f=1-\exp \left(-a t^{b}\right)$ was suggested by Kasaoka [3]. The detailed interpretation for the oxidation rate equation and the calculation methode were reported earlier $[13,14]$.

Fig. 6 presents the results which were calculated via $Y=\ln$ $a+b \mathrm{X}$. In all reacting temperatures, each reaction point obviously stays in the vertical lines of $Y-X$ graphs. Optimization of lines in graphs was tried between reaction paths of 0.2 and 0.8 of the conversion. A correlation
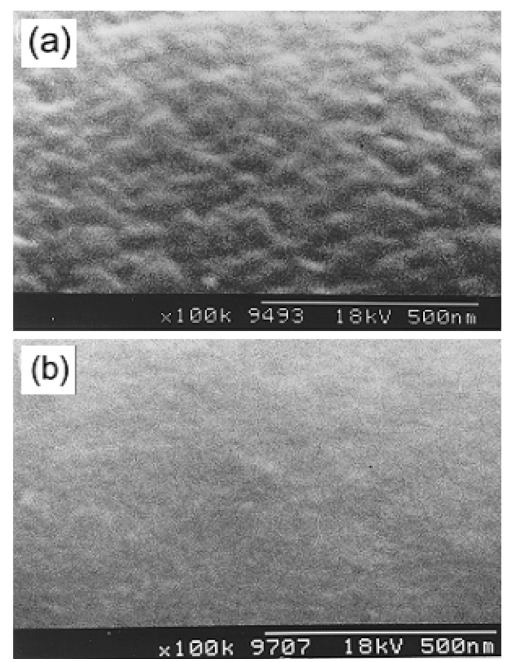

Fig. 4. Highly magnified $(\times 100,000)$ SEM photographs of oxidized IP fiber. The degrees of burn-off are (a) $49 \%$ at $900^{\circ} \mathrm{C}$ and (b) $79 \%$ at $900^{\circ} \mathrm{C}$.
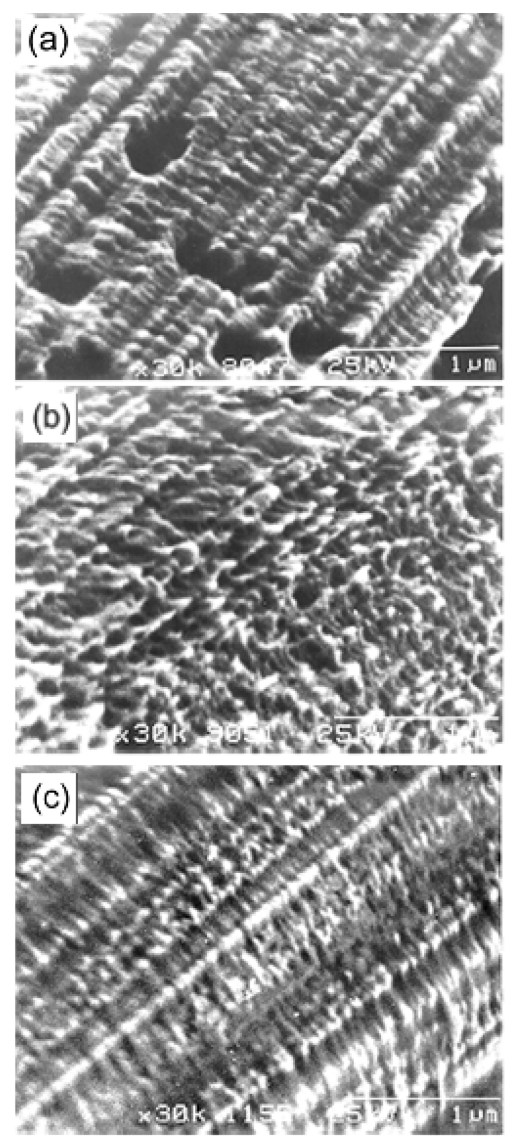

Fig. 5. Highly magnified $(\times 30,000)$ SEM photographs of oxidized $\mathrm{HM}$ fiber. The degrees of burn-off are (a) $47 \%$ at $800^{\circ} \mathrm{C}$, (b) $51 \%$ at $900^{\circ} \mathrm{C}$, and (c) $50 \%$ at $1,100^{\circ} \mathrm{C}$.

coefficient of optimized lines was more than 0.992 . Moreover, $\ln a$ and $b$ gained from $Y=\ln a+b \mathrm{X}$ and a average rate constant $K$ are summarized in Table 3 .

As mentioned in early report, $b$ value of the equation $Y=\ln a+b X$ is important [3,14]. If it is $0<b \leq 1$, oxidation rates decreases as a function of the oxidation reaction proceeds. If it is $b=1$, oxidation rate is generally steady during the whole activation, and activation reaction is the stage controlled by the continuous chemical reaction. If it is $b>1$, $f-t$ curves representing sigmoidal characteristics. This means the reaction rate increases at the core of solid carbon as a function of the oxidation reaction proceeds [3].

As seen in Table 3, Constant $b$ of HM fibers is gained between $1.0285 \sim 1.4268$. The $b$ value decreases as the reaction rates show higher value, and $b$ value of IP fiber is gained between $0.9226 \sim 1.2514$. In the case that the reaction rate is low, as $b$ value of HM fiber is larger than 1, activation reaction with low temperature stimulates active sites during the reaction increasing, and the higher the reacting temperature increases, $b$ value gets closer to 1 so activation in the high temperature does not stimulate active sites during the reaction decreasing. In addition, $b$ value of IP fiber gets closer to 1 at $900^{\circ} \mathrm{C}$ and $1000^{\circ} \mathrm{C}$, so regular activation 

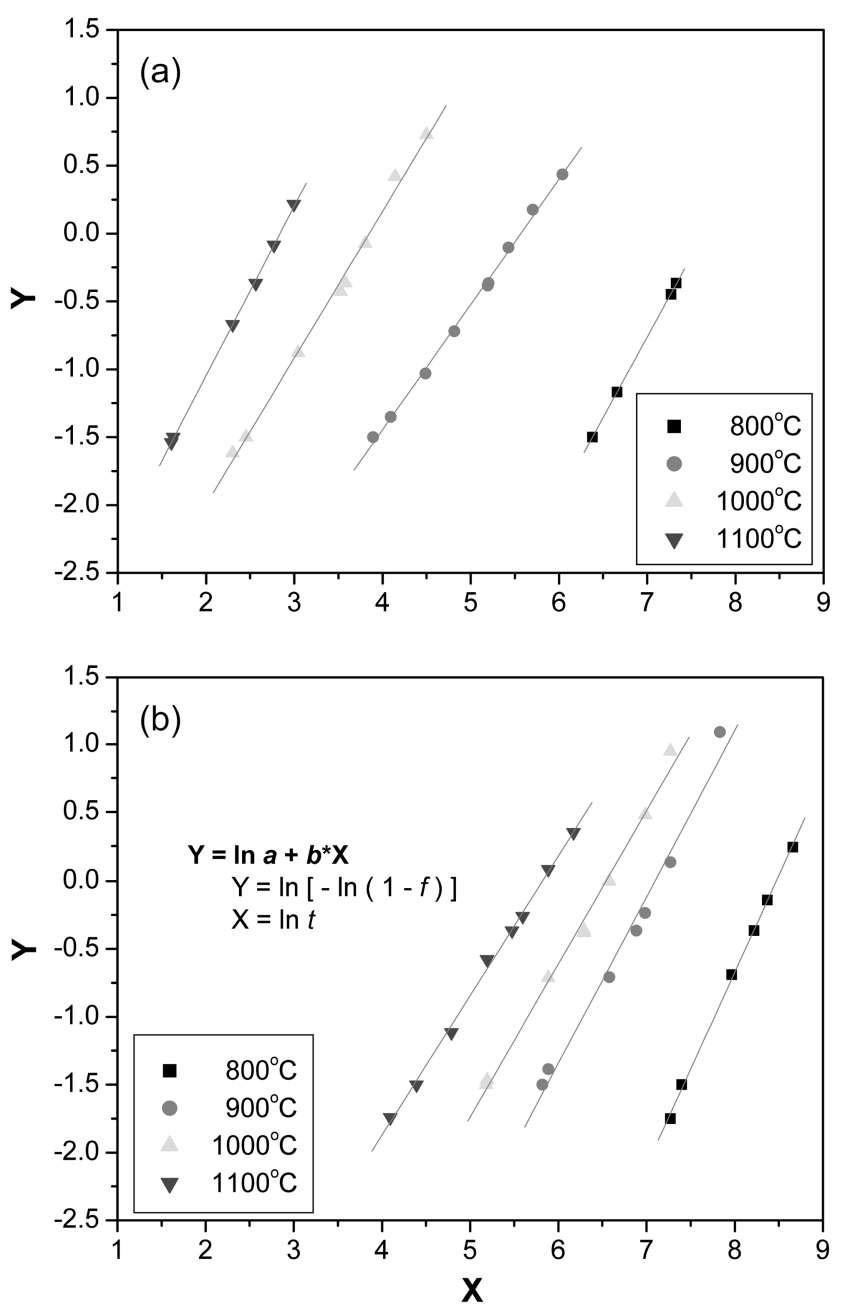

Fig. 6. $Y-X$ plots from $Y=\ln a+b X$ for IP fibers (a) and HM fibers (b).

reaction in active sites take place.

Since reaction of carbon materials to gasification takes place first of all by chemical reaction existing in active sites, when the constant $b$ gained from the above gets closer to 1 , that is, if chemical reaction of regular rate takes place SSA is considered to become larger. Through comparing the $b$ value to the data of the SSA, it is clear that the SSA of the oxidized fiber is much larger in the reacting temperature when $b$ value gets closer to 1 , regardless of kinds of fiber [12]. Also, HM fiber oxidized at high temperatures has more smaller pores than the fiber oxidized at low temperatures in Fig. 5. Therefore there is a possibility that the constant $b$ is related much to development of pores.

\subsection{Oxidation reaction mechanism}

\subsubsection{Activation energy}

Rate constant values according to the conversion ratio at each oxidizing temperature are in Table 4 in order to find out
Table 3. $\ln a, b$ and $K$ Calculated by $Y=\ln a+b X$

\begin{tabular}{cccccc}
\hline Fibers & $\begin{array}{c}\text { Temperature } \\
\left({ }^{\circ} \mathrm{C}\right)\end{array}$ & $\ln a$ & $b$ & $K$ & $r$ \\
\hline $\mathrm{HM}$ & 800 & -12.0875 & 1.4268 & 0.00025 & 0.993 \\
\cline { 2 - 6 } & 900 & -8.7039 & 1.2265 & 0.00095 & 0.992 \\
\cline { 2 - 6 } & 1000 & -7.3781 & 1.1278 & 0.00156 & 0.996 \\
\cline { 2 - 6 } & 1100 & -5.9870 & 1.0285 & 0.00299 & 1.000 \\
\hline IP & 800 & -9.0804 & 1.1180 & 0.00050 & 1.000 \\
\cline { 2 - 6 } & 900 & -5.1373 & 0.9226 & 0.00373 & 0.999 \\
\cline { 2 - 6 } & 1000 & -4.1597 & 1.0806 & 0.02227 & 0.997 \\
\hline
\end{tabular}

Table 4. Reaction Rates as a Function of Conversion

\begin{tabular}{cccccc}
\hline \multirow{2}{*}{ Fibers } & \multicolumn{2}{c}{ Conversion } & \multicolumn{4}{c}{ Reaction rate $(k)$} \\
\cline { 2 - 6 } & $(f)$ & 0.2 & $900^{\circ} \mathrm{C}$ & $1000^{\circ} \mathrm{C}$ & $1100^{\circ} \mathrm{C}$ \\
\hline \multirow{3}{*}{$\mathrm{HM}$} & 0.2 & 0.00019 & 0.00078 & 0.00139 & 0.00249 \\
\cline { 2 - 6 } & 0.5 & 0.00027 & 0.00097 & 0.00159 & 0.00301 \\
\cline { 2 - 6 } & 0.8 & 0.00034 & 0.00112 & 0.00172 & 0.00309 \\
\hline \multirow{2}{*}{ IP } & 0.2 & 0.00045 & 0.00399 & 0.02058 & 0.05420 \\
\cline { 2 - 6 } & 0.5 & 0.00054 & 0.00363 & 0.02239 & 0.06806 \\
\cline { 2 - 6 } & 0.8 & - & 0.00338 & 0.02385 & 0.08082 \\
\hline
\end{tabular}

the reaction mechanism to oxidation according to the conversion ratio, and Arrhenius graph gained by applying natural logarithm to each rate constant is in Fig. 7. In all experimental conditions in Table 4 , the reaction rate increased as the conversion ratio increased.

Activation energy of HM fibers in the Arrhenius graph shows $21.0 \sim 24.7 \mathrm{kcal} / \mathrm{mole}$ and IP fibers in the Arrhenius graph shows $47.6 \sim 51.3 \mathrm{kcal} / \mathrm{mole}$. Oxidation process of IP fiber is controlled by chemical reaction from activation energy, and oxidation process of HM fiber is controlled by the diffusion process through pores on the basis of the concept of the reaction-controlling regime.

Out of several models for interpreting reaction mechanism of gasification of carbon materials using activation energy, Progressive - Conversion Model(PCM) and Shrinking - Core Model(SCM) are well known [4].

Since Shrinking-Core Model(SCM) can be applied to HM fibers, oxidation of fiber proceeds from the surface of the fiber to the center. HM fibers have crystallites very large and dense, and there is not enough space for pores to be created because the number of active sites is limited. Therefore, oxidation of HM fibers are controlled by the fact that pores start developing from the surface, so there could be an explanation that SSA does not largely increase after oxidation.

In the meantime, activation energy of IP fibers are applied to PCM. IP fibers haves small crystallites and loose density, finally giving more active sites for reaction with many pores already inside as well. Therefore, if pores open on the surface in the early stages of activation, it is possible for active sites existing inside the fiber to chemically react. 

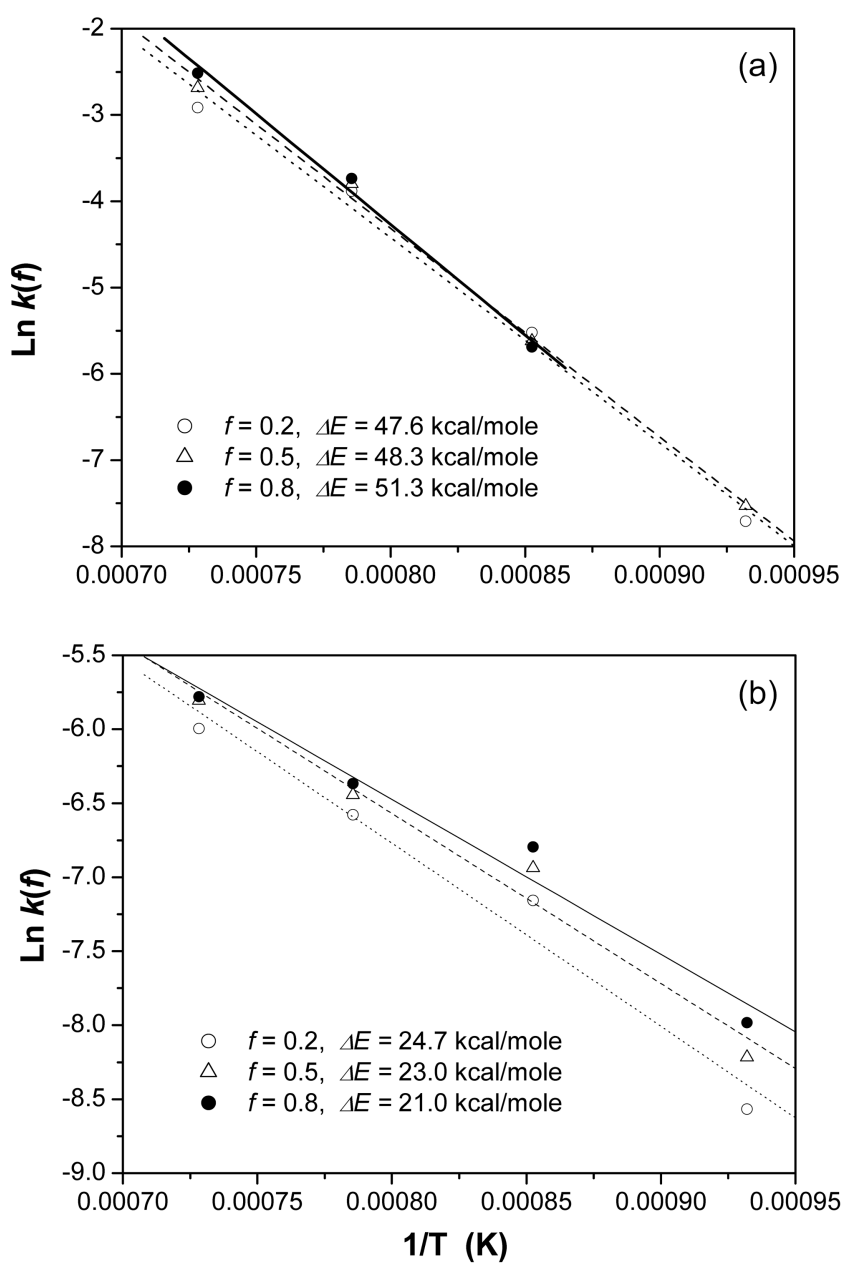

Fig. 7. Changes of Arrehnius plots as a function of conversion.

\subsubsection{Changes of activation energy}

Fig. 8 shows difference in the changes of activation energy according to the conversion ratio between HP fibers and IP fibers. Activation energy data of IP fibers quote from the early report [12]. Activation energy of HM fibers displayed a tendency to decrease from 24.7 to $21.0 \mathrm{kcal} / \mathrm{mole}$ as the conversion increases, and IP fibers, on the other, displayed the increase from 47.6 to $51.3 \mathrm{kcal} / \mathrm{mole}$. To explain the changes of activation energy by means of reaction stages, HM fibers are controlled by diffusion procedures as the conversion increases, and IP fibers are more controlled by chemical reaction.

The reaction between reacting gas and carbon atoms takes place in active sites. Since crystallites of HM fibers are very large, the number of their active sites is limited than IP fibers and the spaces(that is, pores) in between crystallites are very narrow [15]. Besides, mesophase carbon fibers have a inhomogeneous structures that show a difference in graphite and density according to part of fiber while being manufactured $[8,15,17]$. So, reaction of the initial oxidation, considering the overall structure of the fiber, is likely to take

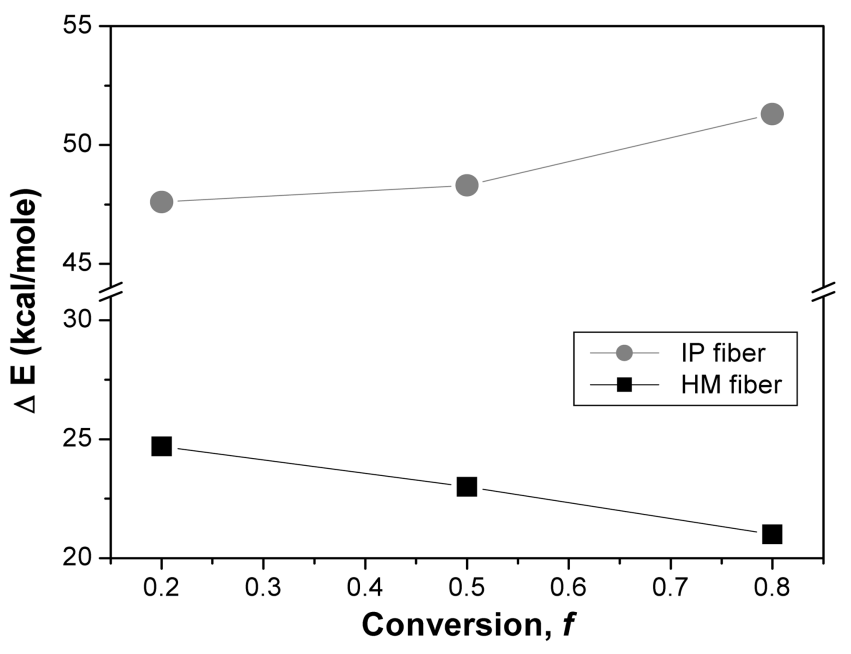

Fig. 8. Changes of the activation energy as a function of conversion.

place at a less dense area. The following oxidation reaction is more easily taken place after opening pores from fiber surface to inside.

The number of active sites are proportional to the number of carbon atoms at the crystallite-ends. If the crystallites are small, there are more active sites. This means that the number of active sites exposed to the crystallite-ends increase as activation reaction proceeds. For the IP fibers in Fig. 8, it seems that activation energy increases as the conversion is that the number of active sites increases. However the active site increase, the activation reaction is not more easy as residual carbon remains very small amount for oxidation.

Even though those two kinds of fiber do not have a large difference in changes of activation energy and if there is a little error, it seems that the difference in energy change of two kinds of fibers is related to the characteristics of pores developed after oxidation reaction. Therefore the reason that changes of oxidation mechanism show the opposite according to the conversion is that pore developments during activating are different due to the structural difference of two kinds of fiber.

\section{Conclusion}

High modulus pitch based carbon fibers were exposed to oxidation in carbon dioxide. After activation energy was gained according to the conversion at each reacting temperature, the value of SSA and the surface morphology was compared, and the reaction mechanism of oxidation affecting development of pores of carbon fibers was examined to get the results like the following.

Activation energy of HM fibers decreases from $24.7 \mathrm{kcal} / \mathrm{mole}$ to $21.0 \mathrm{kcal} / \mathrm{mole}$ with increasing conversion 0.2 to 0.8 and 
oxidation reaction is limited by diffusion through pore as the conversion increases.

The constant $b$ of the reaction equation used in this study is considered very useful. It observed that the constant $b$ gets closer to 1, the SSA gets higher. Therefore, it is believed that the SSA of the fiber oxidized at a certain temperature increase when $b$ value gets nearer 1 regardless of kinds of fibers.

\section{References}

[1] Mahajan, O. P.; Yarzab R.; Walker Jr. P. L. Feul, 1978, 57, 643.

[2] Sanchez, A. R.; Elguezabal, A. A.; Torre Saenz, L. L. Carbon, 2001, 39, 1367.

[3] Kasaoka, S.; Sakata, Y.; Kayano, S.; Masuoka, Y. Int. Chem. Eng. 1983, 23, 477.

[4] Hu, Y. Q.; Nikzat, H.; Nawata, M.; Kobayashi, N.; Hasatani, M. Feul, 2001, 80, 2111.
[5] Rafsanjani, H. H.; Jashidi, E.; Rostam-Abadi, M. Carbon, 2002, 40, 1167.

[6] Lafdi, K.; Bonnamy, S.; Oberlin, A. Carbon, 1992, 30, 533.

[7] Ismail, M. K. Carbon, 1991, 29, 777.

[8] Li, T.; Zheng, X. Carbon, 1995, 33, 469.

[9] Tanabe, Y.; Utasunomiya, M.; Ishibashi, M.; Kyotani, T.; Kaburagi, Y.; Yasuda, E. Carbon, 2002, 40, 1.

[10] Matsumura, Y.; Xu, X.; Antal, M. J. Jr. Carbon, 1997, 35, 819.

[11] Tomlinson, J. B.; Freeman, J. J.; Sing, S. W.; Theocharis, C. R. Carbon, 1995, 33, 789.

[12] Roh, J. S. Carbon Science, 2005, 6, 51.

[13] Roh, J. S. Carbon Science, 2003, 4, 185.

[14] Roh, J. S.; Suhr, D. S. Carbon Science, 2004, 5, 51.

[15] Donnet, J. B.; Bansal, R. C. "Carbon Fibers", 2nd ed., Marcel Decker, inc. 1990, 128.

[16] Marsh, H.; Reinoso, F. R. "Science of Carbon Materials", Universidad de Alicante, Alfredo Candela, 2000, 8.

[17] Zheng, G.; Sano, H.; Suzuki, K.; Kobayashi, K.; Uchiyama, Y.; Cheng, H. M. Carbon, 1999, 37, 2057. 